\title{
Encapsulación de quercetina en nano y micro-emulsiones alimenticias
}

\author{
Xavier, M. P. ${ }^{(1) *}$, Miraballes, I. ${ }^{(3)}$, Pardo, H. ${ }^{(3)}$, Mombrú, A. ${ }^{(3)}$, López, T. ${ }^{(1,2)}$, Medrano, C. A. ${ }^{(1)}$ \\ (1) Departamento de Ciencia y Tecnología de los Alimentos, Facultad de Química, Universidad de la República, Uruguay \\ ${ }^{(2)}$ Centre Especial de Recerca, Planta de Tecnología de los Alimentos, Facultad de Veterinaria, Universidad Autónoma \\ de Barcelona - ${ }^{(3)}$ Polo Tecnológico de Pando, Facultad de Química, Universidad de la República.
}

Contacto: pazxavier@gmail.com

Recibido: 30/06/2011 - Aprobado: 26/10/2011

\section{Resumen}

El objetivo de este estudio fue desarrollar, caracterizar y comparar nano y microemulsiones estables incorporando quercetina como compuesto bioactivo. Se estudiaron emulsiones aceite:agua con beta-lactoglobulina, utilizando dos procesos de elaboración: homogeneizador de alta velocidad (Ultra-turrax) y homogeneizador de alta presión (HPH, Emulsiflex-C5, 500-1200bar). Se evaluó la variación en la concentración de emulsionante, fracción volumétrica de aceite e incorporación de hidrocoloides. La formación y la estabilidad de las emulsiones se analizaron por su perfil de tamaño de gota (MalvernZetasizer Nano ZS) y constantes cinéticas de desestabilización obtenidas a partir del estudio de la retro dispersión de la luz en el tiempo (Turbiscan-Classic). Se determinó que utilizando el Ultra-turrax se logra formar nanoemulsiones con mayor estabilidad a una concentración de $2.2 \%(\mathrm{~m} / \mathrm{v})$ de proteína y $0.2 \%(\mathrm{~m} / \mathrm{v})$ de goma guar, mientras que utilizando HPH con una concentración de $1.5 \%(\mathrm{~m} / \mathrm{v})$ de beta-lactoglobulina y sin necesidad de hidrocoloides se logra una emulsión de adecuado tamaño de gota $(\mathbf{d}<500 \mathrm{~nm})$ y mayor estabilidad $(\alpha<0.05)$. Las nanoemulsiones elaboradas con HPH presentaron mayor porcentaje de encapsulación de quercetina.

Palabras clave: Antioxidante, beta lactoglobulina, vehículo alimentario.

\begin{abstract}
The objective of this study was to develop, characterize and compare quercitin loaded nano and microemulsions systems. Oil in water emulsions were prepared using a high speed homogenizer (Ultra Turrax) and a high pressure homogenizer (HPH, Emulsiflex-C5, 500-1200bar). Hydrocolloids incorporation, Beta-lactoglobulin concentration and volumetric fraction variation were evaluated. Emulsifyng capacity and stability were analyzed by light backscattering (Turbiscan Classic) and droplet profile analysis (Malvern Zetasizer Nano). Using the Ultra Turrax optimum conditions for stability with a suitable particle size $(d<500 \mathrm{~nm})$ were achieved with a concentration of $2.2 \%(\mathrm{~m} / \mathrm{v})$ protein and between $0.1-0.2 \%(\mathrm{~m} / \mathrm{v})$ of guar gum, while using HPH emulsions showed higher stability $(\alpha<0.05)$ achieved at a concentration of $1.5 \%$ of Beta-lactoglobulin $(\mathrm{m} / \mathrm{v})$ and without using hydrocolloids. Emulsions made with high pressure achieved greater percentage of quercetin encapsulation.

Keywords: Antioxidants, Beta-Lactoglobulin, food vehicles.
\end{abstract}

\section{Introducción}

Existen ciertas características en los alimentos que le confieren valor agregado y que son valoradas positivamente por los consumidores, tales como la escasa manipulación, la ausencia de aditivos o el empleo de aditivos naturales, la conservación de las propiedades nutricionales y de las cualidades beneficiosas para la salud, entre otras. La incorporación de compuestos bioactivos en sistemas alimentarios puede proporcionar un método simple para desarrollar alimentos funcionales innovadores, que pueden aportar beneficios fisiológicos o reducir la disposición a la enfermedad.

La problemática que existe en algunos casos es que los compuestos activos contenidos en los productos no llegan a aportar el debido beneficio al organismo por su degradación durante la elaboración, almacenamiento $\mathrm{y} / \mathrm{o}$ transporte del producto, o porque el cuerpo humano los destruye antes de que puedan ejercer su efecto. Las nanotecnologías pueden ayudar a mejorar la liberación, absorción y/o protección de estos ingredientes, permitiendo al compuesto realizar su beneficio en el lugar adecuado, aumentando así la calidad nutricional del alimento sin otorgarle propiedades sensoriales indeseables (Augustin, 2003). Si bien la nanotecnología se encuentra actualmente implantada en la industria farmacéutica y cosmética mundial, su aplicación al dominio alimentario ha sido más lenta, ya que la variedad de compuestos a utilizar se ve reducida y es necesario desarrollar nuevos polímeros y/o ingredientes de calidad alimentaria seguros para su ingesta en la dieta.

Dicha tecnología permite lograr, por ejemplo, nanoemulsiones, emulsiones de tamaño de gota muy pequeño $(20-500 \mathrm{~nm}$ ) (Solans et al., 2003), que pueden ser contenedores y transportadores de moléculas con principios activos (Tadros et al., 2004) y presentan mayor estabilidad que las emulsiones de tamaño de gota mayor (Lizarraga et al., 2008). Para lograr los nanovehículos mencionados se cuenta, entre otros métodos, con la alta presión de homogeinización (HPH), una tecnología basada en los principios de la homogeinización convencional (18-20MPa), pero que permite alcanzar presiones muy superiores de hasta 200-220MPa.

Resulta de suma utilidad incorporar en los alimentos consumidos habitualmente por la población, como es el caso de 
los productos lácteos, compuestos bioactivos que no se encuentren o se degraden antes de ejercer su beneficio. La quercetina (Q) (3,3'4',5,7-pentahidroxiflavona) es un flavonoide presente en frutas y verduras con fuerte carácter antioxidante. La capacidad antioxidante total de la $\mathrm{Q}$ medida como actividad antioxidante equivalente en Trolox (TEAC) es de 4,7 mM, lo que representa un valor cinco veces superior a los cuantificados para las vitaminas E y C (Rice-Evans y Miller, 1998; Martínez-Florez et al., 2002).

Los flavonoides ingeridos a través de la dieta se encuentran abundantemente en forma 3-O-glicosilada. El glicósido más abundante de la $\mathrm{Q}$ es la rutina, quercitina unida mediante enlace O-glicósidico a un disacárido glucosa-ramnosa. Dicha glicosilación aumenta su hidrosolubilidad, pero disminuye su poder antioxidante (Heim et al., 2002), por lo que sería provechoso poder hacer llegar al organismo la forma aglicona (quercetina) de este flavonoide. Los procesos de inclusión o encapsulación de estos compuestos bioactivos dentro de matrices deben ser estudiados, caracterizados y puestos a punto.

El objetivo del trabajo se orienta a la producción y comparación de nano y microemulsiones que puedan reintroducirse posteriormente en matrices alimentarias, con la incorporación de quercetina como compuesto bioactivo y utilizando proteínas lácteas para su estabilización. Este desarrollo podrá contribuir a la creación de alimentos de alto valor agregado.<smiles>O=c1c(O)c(-c2ccc(O)c(O)c2)oc2cc(O)cc(O)c12</smiles>

Figura 1. Estructura química de la quercetina.

\section{Materiales y Métodos}

\section{Preparación de las emulsiones}

\section{Preparación de emulsiones con homogeneizador} de alta velocidad

Se prepararon emulsiones aceite en agua variando la fracción volumétrica ( $\varphi=$ volumen aceite/volumen emulsión) y la concentración de Beta-lactoglobulina (Blg). Se utilizaron valores de $\varphi$ de 0,$05 ; 0,25$; 0,4; 0,5 y 0,75 y de Blg de $1,3,5,7,8$ y $10 \mathrm{mg} / \mathrm{ml}$ en disolución de fosfato de sodio $0,1 \mathrm{M}, \mathrm{pH} 7,4$. Algunas de las emulsiones contaron con el agregado de pectina (de alto y bajo metoxilo) y otras de goma guar disueltas en igual solución que la proteína, en concentraciones de 0,$5 ; 0,7$ y de 0,$05 ; 0,1 ; 0,15 ; 0,2 \mathrm{mg} / \mathrm{mL}$, respectivamente. Las emulsiones fueron preparadas a temperatura ambiente con un homogeneizador Ultra-turrax T25 (IKA-Labortechnik, GmbH \& Co., Staufen, Alemania) (UT), utilizando el accesorio dispersante S 25 $\mathrm{N}-10 \mathrm{G}$ a una velocidad de $20.000 \mathrm{rpm}$ durante un minuto. Previamente a la homogeinización se incorporaron a la fase oleosa $300 \mu \mathrm{L}$ de una solución de Q en etanol de concentración $5 \mathrm{mg} / \mathrm{mL}$ cada $100 \mathrm{~mL}$ de dicha fase.

\section{Preparación de emulsiones por alta presión de homogeinización}

Se prepararon emulsiones aceite:agua 50:50 con Blg como emulsionante en concentraciones 0,$5 ; 1,5 ; 2,5$ y $3,5 \% \mathrm{~m} / \mathrm{v}$. La proteína fue dispersada en solución de fosfato de sodio $0,1 \mathrm{M} \mathrm{pH} 7,4$. La preparación aceite-agua fue premezclada en un agitador de paleta durante 1 minuto previo a su pasaje por el equipo de HPH (Emulsiflex C5, Avestin). Se experimentaron dos rangos de presión (500-800bar) y (800-1200bar) y cada emulsión fue reciclada cinco veces. De cada uno de los rangos se analizaron muestras de los ciclos 1,3 y 5 . Las emulsiones ingresaron al equipo a $25^{\circ} \mathrm{C}$ (temperatura de entrada del primer ciclo) y en los siguientes ciclos se determinaron la temperatura de entrada y salida.

Previo a la homogeinización se incorporaron a la fase oleosa $300 \mu \mathrm{L}$ de una solución de Q en etanol de concentración $5 \mathrm{mg} / \mathrm{mL}$ cada $100 \mathrm{~mL}$ de la fase.

\section{Análisis de la capacidad para formar y estabilizar emulsiones aceite-agua}

La estabilidad global de las emulsiones se analizó mediante un analizador óptico vertical (Turbiscan Classic MA 2000, Formulaction, Toulouse, France) (Palazolo et al., 2004; Palazolo et al., 2005). Las emulsiones recientemente preparadas se colocaron en una celda cilíndrica de vidrio $(80 \mathrm{~mm})$ para registrar el perfil de retrodispersión (RD \%) o "Back Scattering" (\%BS) en función de la altura en la celda. Se realizó el estudio de las cinéticas de cremado y desestabilización de las emulsiones mediante la medida de BS cada un minuto durante un período de 60 minutos y una medida a las $24 \mathrm{hs}$. En el caso de las emulsiones obtenidas con HPH el estudio se continuó con medidas a los 5, 15 y 30 días. El estudio se realizó en la parte inferior $(8 \mathrm{~mm})$ del tubo. Se utilizaron modelos cinéticos planteados por Panizzolo (2005).

\section{Determinación del tamaño promedio de gota en las emulsiones}

La distribución del tamaño de partícula se determinó por difracción de luz láser y dispersión de luz polarizada mediante el uso de un analizador de partículas Coulter Counter Multisizer (Coulter Electronics Ltd) (Anton et al., 2002), que mide en un rango de 0.04 $\mu \mathrm{m}$ hasta $2000 \mu \mathrm{m}$, y por dispersión de luz láser mediante Malvern Zetasizer NanoZS, que mide en el rango de $0.1 \mathrm{~nm}$ y $6 \mu \mathrm{m}$, en el caso de las emulsiones obtenidas mediante UT y HPH, respectivamente. Las emulsiones analizadas fueron preparadas según lo descripto en el apartado 1, Preparación de las emulsiones. Se utilizaron también como parámetros de comparación el diámetro de Sauter $\left(D_{3,2}=\sum \mathrm{d}^{3} / \mathrm{d}^{2}\right)$ y el diámetro de Broucker $\left(\mathrm{D}_{4,3}=\sum \mathrm{d}^{4} / \mathrm{d}^{3}\right)$

\section{Determinación del porcentaje de encapsulación de Quercetina (Q)}

La determinación del porcentaje de encapsulación del compuesto bioactivo en las emulsiones se realizó mediante cromatografía líquida de alta resolución (HPLC). Para ello se usó un equipo de HPLC Shimadzu con detector UV Shimadzu SPD-20A y bomba Shimadzu LC-10AT, utilizando Acetonitrilo:Agua 40:60 como fase móvil (Kumari et al., 2010) y columna Jupiter C18 de tamaño $250 \mathrm{~mm}$ de largo, 4,6 mm de diámetro y $5 \mu \mathrm{m}$ de tamaño de poro. Se utilizó un flujo de $1 \mathrm{~mL} / \mathrm{min}$. La curva patrón se realizó con soluciones de Q en etanol abarcando concentraciones desde 0.001 a $0.1 \mathrm{mg} / \mathrm{ml}$, la 
linealidad se mantuvo entre 0.001 y $0.05 \mathrm{mg} / \mathrm{ml}$ con $\mathrm{R}^{2}=0.9991 . \% \mathrm{Q}$ encapsulada $=($ Qi-Qs $) /$ Qi x 100 con Qi: masa de Q incorporada en la emulsión y Qs: masa de Q en el sobrenadante.

Para el análisis, las emulsiones fueron centrifugadas a $3000 \mathrm{~g} \mathrm{y}$ $4{ }^{\circ} \mathrm{C}$ durante 2 y 5 horas en el caso de emulsiones obtenidas en UT y $\mathrm{HPH}$, respectivamente, logrando así un cremado sin rompimiento de la emulsión. La emulsión fue congelada para obtener la fase acuosa libre de la fase crema. Luego la fase acuosa fue diluida (1/5) en etanol absoluto, de forma de precipitar la proteína, y posteriormente centrifugada a $12000 \mathrm{~g}$ por 15 minutos a $4{ }^{\circ} \mathrm{C}$. El sobrenadante obtenido fue el analizado.

\section{Análisis estadístico}

El tratamiento estadístico de datos se efectuó en todos los casos mediante análisis de la varianza (ANOVA) con $\alpha=0,05 \mathrm{y}$ la comparación de medias por la prueba de mínimas diferencias significativas (LSD) con $\alpha=0,05$, con el programa Statgraphics plus 7.0.

\section{Resultados y Discusión}

Se determinó que las emulsiones de $\varphi=0.5$ sin hidrocoloides realizadas con ultraturrax presentan mayor estabilidad en comparación a los demás $\varphi$ estudiados. Se comprobó, tal como era esperado, que los estabilizantes experimentados (pectina, GG) aumentan la estabilidad de la emulsión frente a la floculación y al cremado. No hubo diferencia en los resultados de estabilidad al utilizar pectina de alto $(\mathrm{HM}=55)$ o de bajo ( $\mathrm{LM}=32)$ metoxilo. Todas las condiciones ensayadas en emulsiones obtenidas con el UT con o sin pectina presentaron una cinética de desestabilización del tipo sigmoidea $(\mathrm{S})$, hiperbólica $(\mathrm{H})$ o una conjunción de ambas (C). Sin embargo, cuando se utilizó un hidrocoloide de mayor peso molecular, como es el caso de goma guar, aumentó significativamente la estabilidad; en el caso de la emulsión aceite agua de $\varphi=0.4$ con $2.2 \%(\mathrm{~m} / \mathrm{v})$ de Blg y $0.2 \%(\mathrm{~m} / \mathrm{v})$ de goma guar (GG) se logró una cinética de desestabilización lineal dentro de las 24 horas, comparable a las cinéticas obtenidas para las emulsiones tratadas con HPH presentadas y discutidas más adelante. Este tipo de cinética puede deberse al aumento de la viscosidad de la fase continua que provocaría una disminución en la velocidad de cremado, según la ley de Stokes.

Respecto al tamaño promedio de gota, fue menor significativamente cuando se manejaron concentraciones de $2 \%$ o $3.5 \%(\mathrm{~m} / \mathrm{v})$ de proteína y $0.5 \%(\mathrm{~m} / \mathrm{v})$ de pectina. Comparándolas con las emulsiones de iguales características sin el agregado de pectina se observa (Figura 2) que el diámetro promedio al contener $0.5 \%$ de pectina es cercano a los $100 \mathrm{~nm}$, mientras que en emulsiones sin agregado del hidrocoloide ronda en los $450 \mathrm{~nm}$. El valor de $\mathrm{D}_{3,2}$ es similar en ambas, no obstante el de $\mathrm{D}_{4,3}$ es levemente mayor en presencia de la pectina, es decir que, a pesar de que el tamaño promedio de partícula se redujo y casi el $100 \%$ de las partículas tienen un diámetro menor a $500 \mathrm{~nm}$, las pocas $(<1 \%)$ restantes representan alrededor del $95 \%$ del volumen. Pese a que un aumento en el valor del $\mathrm{D}_{4,3}$ podría provocar un aumento en la velocidad de cremado, la mayor estabilidad apuntada anteriormente -durante los primeros 60 minutos luego de formada la emulsión-, puede deberse al incremento de la viscosidad de la fase continua. La emulsión preparada con GG descripta mostró (Figura 2) un diámetro promedio menor al de la emulsión sin estabilizantes y mayor al de la emulsión con pectina ya analizada, en tanto que su D 4,3 fue menor al de estas emulsiones. Dicho valor, junto con el aumento de la viscosidad de la fase continua, puede explicar su mayor estabilidad.
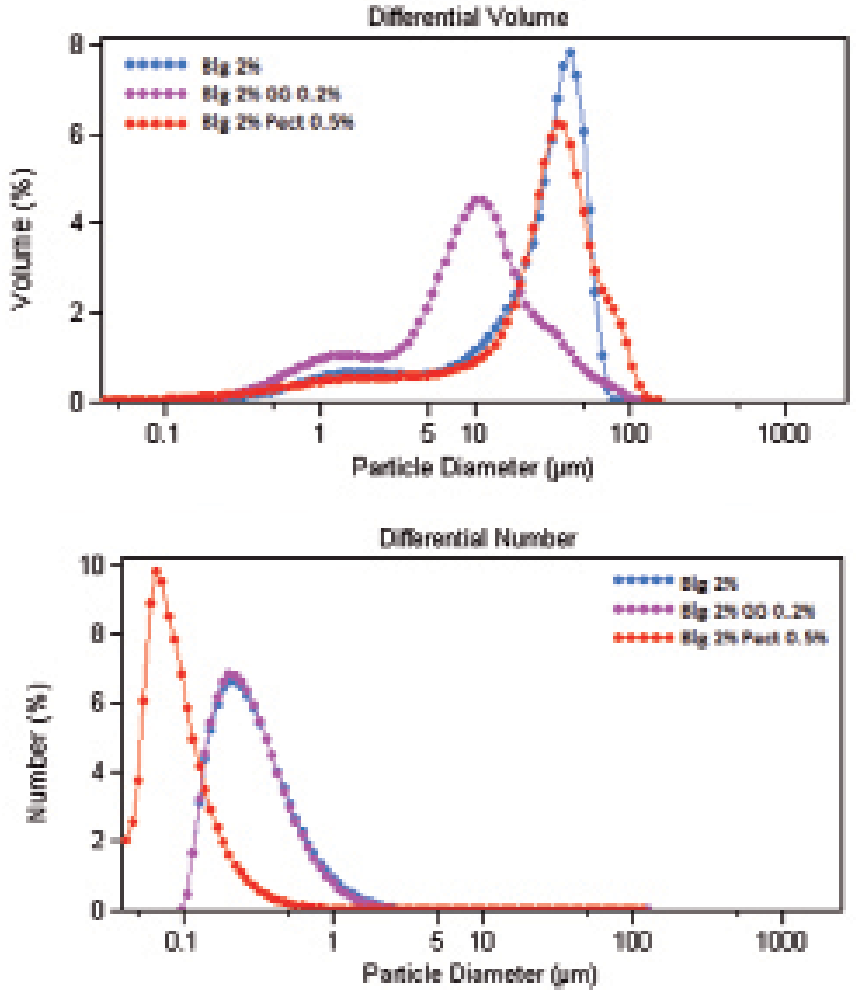

Figura 2. Distribución de tamaño en volumen (a) y en número (b) de

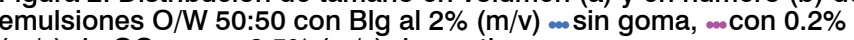
$(\mathrm{m} / \mathrm{v})$ de $G G$, coccon $0.5 \%(\mathrm{~m} / \mathrm{v})$ de pectina.

Dentro de las emulsiones tratadas con HPH se observó que para una concentración de proteínas de $0.5 \%(\mathrm{~m} / \mathrm{v})$, todos los ciclos estudiados ( 1,3 y 5$)$ pasan de presentar una cinética lineal de desestabilización en la primera hora a una $\mathrm{S}, \mathrm{H}$ o $\mathrm{C}$ a las 24 horas. Al aumentar la concentración a 1.5 y $2.5 \%$ se mantiene la cinética lineal hasta los 5 días en el caso del primer reciclado, hasta los 15 días en el caso de tres pasajes y hasta los 30 días cuando es reciclada 5 veces. En la Fig. 3A) se puede observar las emulsiones con $1.5 \%$ de Blg obtenidas con 1,3 y 5 ciclos, el control del equipo (pasaje por el equipo de HPH a presión atmosférica) y la emulsión sin pasaje por el equipo de HPH luego de un mes. En la Fig. 3B) y 3C) se muestran sus cinéticas obtenidas con el turbiscan.

Una estabilidad aún mayor presentan las emulsiones con $3.5 \%$ de $\mathrm{Blg}$, logrando mantener una cinética de desestabilización lineal por 15 días en el primer ciclo y por más de 45 días en los ciclos 3 y 5 , sin que se hallara diferencia de estabilidad entre los distintos rangos de presiones estudiados (500-800 bar; 800-1200 bar). El aumento de estabilidad al aumentar el número de ciclos se debe a la progresiva reducción de tamaño que se muestra en la Figura 4, sobre todo de las partículas más grandes, principales causantes de la rápida desestabilización. La mayor estabilidad de las emulsiones tratadas con HPH respecto a las obtenidas con UT se puede corresponder, además de al menor tamaño logrado en las primeras, a la conformación del emulsificante; en el caso de las proteínas séricas éstas se pueden ver afectadas por la HPH y sufrir desnaturalización cuando son tratadas con presiones superiores a los $200 \mathrm{MPa}$ (Gracia et al., 2008)

Comparando los tamaños de partícula de ambos métodos es posible observar que al utilizar HPH el $\mathrm{D}_{3,2}$ disminuye entre 4 y 5 veces y el $\mathrm{D}_{4,3}$ lo hace en por lo menos 10 veces. Esta disminución de tamaño es un reflejo del mecanismo del homogeneizador de HPH, el cual consiste en un generador de alta presión ensamblado a una válvula diseñada para resistir la aplicación de muy altas presiones. Como consecuencia 


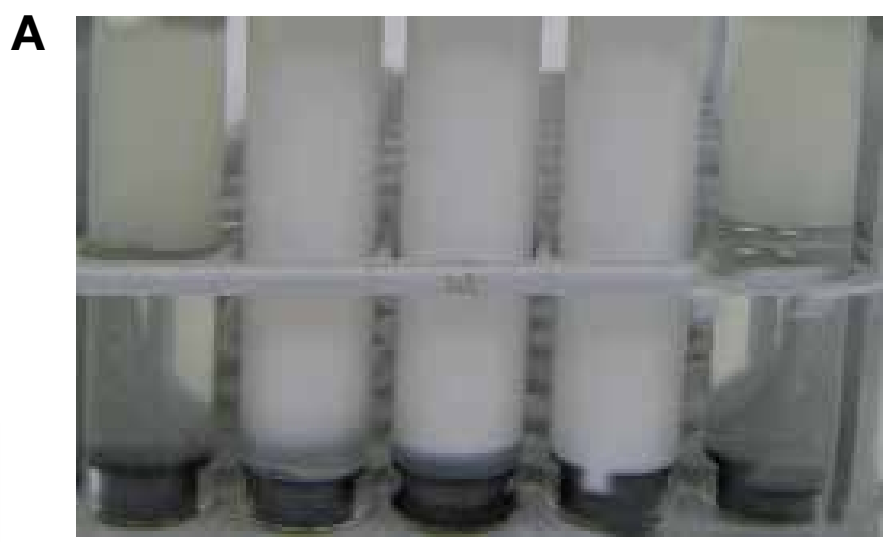

B


Figura 3. A) Emulsión 50:50 con Blg al 1.5\% con un mes de antigüedad (a) Control (P atm), (b) ciclo 1, (c) ciclo 3, (d) ciclo 5, (e) control equipo. B) cinética lineal que presenta c. y b. C) cinética sigmoidea que presenta a.

(a)


(b)
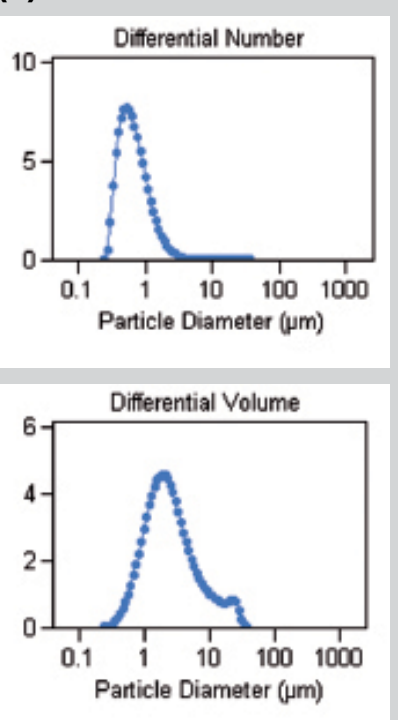

se producen fuerzas de cizalla, cavitación, turbulencia y coalescencia en la superficie del fluido, que pueden causar, entre otros efectos, la ruptura de las gotas de grasa en el caso de una emulsión aceite en agua $\mathrm{O} / \mathrm{W}$, aumentando su estabilidad (Desrumaux y Marcand, 2002)

El porcentaje de encapsulación de quercetina en emulsiones obtenidas mediante enfoque tradicional fue de 12 , mientras que en emulsiones con HPH (Blg 1.5\% tercer ciclo, 800-1200bar) resultó de 40 , lo cual se puede deber al tamaño de partícula más pequeño obtenido con HPH. El consiguiente aumento de la interfase entre las partículas y su medio circundante puede ser favorable para aumentar la biodisponibilidad de compuestos (Chen et al., 2006).

\section{Conclusión}

Utilizando el método tradicional de obtención de emulsiones, la mayor estabilidad (24 horas) se logra con una concentración de $2.2 \%$ $(\mathrm{m} / \mathrm{v})$ de proteína y $0.2 \%(\mathrm{~m} / \mathrm{v})$ de goma guar. Utilizando HPH $(800-$ 1200 bar) con una concentración de $1.5 \%(\mathrm{~m} / \mathrm{v})$ de beta-lactoglobulina y sin necesidad de hidrocoloides es posible lograr emulsiones con una estabilidad aceptable de 5 días con un ciclo y de 15 días al reciclarse 3 y 5 veces. Éstas tienen un $99 \%$ de sus partículas de diámetro menor a $500 \mathrm{~nm}$. Las nanoemulsiones elaboradas con HPH (1.5\% BLG) presentaron mayor porcentaje de encapsulación de quercetina.

\section{Reconocimientos}

Los autores del artículo agradecen la financiación otorgada por la Comisión Sectorial de Investigaciones Científicas (Udelar) y el comité ECOS-SUD-Proyecto 008B01.

\section{Referencias}

- ANTON, M.; BEAUMAL, V.; BROSSARD, C.; LLAMAS, G.; LE DENMAT, M. Droplet flocculation and physical stability of oilin-water emulsions prepared with hen egg yolk. En: ANTON, M. Food emulsions and dispersions. Kerala: Research Signpost, 2002. pp.15-28.

(c)
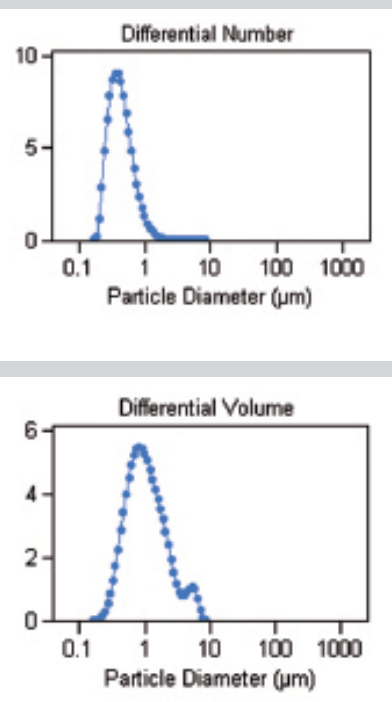

(d)
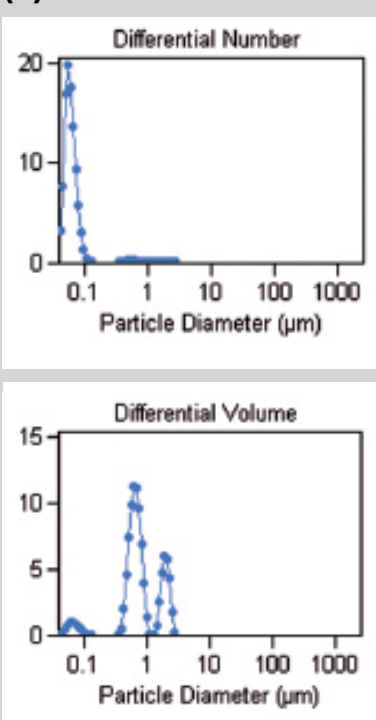

Figura 4. Gráficos de distribución de número y volumen de tamaño de gota de emulsión 50:50, Blg $3.5 \%$ medidos inmediatamente luego de formada la emulsión en analizador óptico Coulter. (a) emulsión control (Patm); (b) ciclo 1 (500-800 bar); (c) ciclo 3 (500-800 bar); (d) ciclo 5 (500-800 bar). 
- AUGUSTIN, M. A. The role of encapsulation in the development of functional dairy foods. En: Australian Journal of Dairy Science and Technology. 2003, 58:156-160.

- CHEN, L.; REMONDETTO, G.E.; SUBIRADE, M. Food proteinbased materials as nutraceutical delivery systems. En: Trends Food Science and Technolgy. 2006, 17:272-83.

- DESRUMAUX, A.; MARCAND, J. Formation on sunflower oil emulsions stabilized by whey protein with high-pressure homogenization: effect of pressure on emulsion characteristics. En: International Journal of Food Science and Technology. 2002, 37:263-269.

- GRACIA-JULIA. A.; RENE, M.; CORTES-MUÑOZ, A.; PICART, L.; LOPEZ-PEDEMONTE, T.; CHEVALIER, D.; DUMAY, E. Effect of dynamic high pressure on whey protein aggregation: a comparison with the effect of continuous short-time thermal treatments. En: Food Hydrocolloids. 2008, 22(6):1014-1032.

- HEIM, K.E.; TAGLIAFERRO, A.R.; BOBILYA, D.J. Flavonoid antioxidants: chemistry, metabolism and structure-activity relationships. En: J.Nutr.Biochem. 2002, 13:572-584.

- KUMARI, A.; YADAV, S. K.; PAKADE, Y.; KUMAR, V.; SINGH, B.; CHAUDHARY, A.; YADAV, S. C. Nanoencapsulation and characterization of Albizia chinensis isolated antioxidant quercetin in PLA nanoparticles. En: Colloids and Surfaces B: Bioenterfaces. 2010, 82:224-332.

- LIZARRAGA, M.S.; PAN, L.G.; AÑOB, M.C.; SANTIAGO, L.G. Stability of concentrated emulsions measured by optical and rheological methods. Effect of processing conditions - I. Whey protein concentrate. En: Food Hydrocolloids. 2008, 22:868-878

- MARTÍNEZ-FLÓREZ, S.; GONZÁLEZ-GALLEGO, J.; CULEBRAS, J. M.; TUÑÓN, M. J. Los flavonoides: propiedades y acciones antioxidantes. En: Nutr. Hosp. 2002, XVII(6):271-278.

- PAlazOlO, G. G.; SORGENTINI, D. A.; WAGNER, J. R. Emulsifying properties and surface behavior of native and denatured whey soy proteins in comparison with other proteins.Creaming stability of oil-in water emulsions. En: Journal of the American Oil of Chemists'Society. 2004, 81:625-632.

- PAlazolo, G. G.; SORGENTINI, D. A.; WAGNER, J. R. Coalescence and flocculation in $\mathrm{o} / \mathrm{w}$ emulsions of native and denatured whey soy proteins in comparison with soy protein isolates. En: Food Hydrocolloids. 2005, 19:595-604.

- PANIZZOLO, L. A. Modificación de proteinas por vía enzimática. Análisis de la relación estructura funcionalidad de los productos de hidrólisis. Montevideo: Facultad de Química, 2005.

- RICE-EVANS, C.A.; MILLER, N.J. Structure-antioxidant activity relationships of flavonoids. En: RICE-EVANS, C.A.; PACKER, L. Flavonoids in health and disease. New York: Marcel Dekker, 1998. pp. 541

- SOLANS, C.; ESQUENA, J.; FORGIARINI, A.M.; USÓN, N.; MORALES, D.; IZQUIERDO, P.; AZEMAR, N.; GARCIACELMA, M.J. Nano-emulsions: formation, properties and applications. En: Surfactant Sci Series. 2003, 109:525-54.

- TADROS, T.; IZQUIERDO, P.; ESQUENA, J.; SOLANS, C. Formation and stability of nano-emulsions. En: Adv Colloid Interface Sci. 2004, (108-109):303-318 